\title{
NONLINEAR MODELLING AND CONTROL OF A LONG RIVER STRETCH
}

\author{
X. Litrico*, J.-B. Pomet ${ }^{\dagger}$ \\ * Cemagref, UR Irrigation, B.P. 5095, 34033 Montpellier Cedex 1, France, e-mail: xavier.litrico@cemagref.fr \\ ${ }^{\dagger}$ INRIA, Projet MIAOU, B.P. 93, 06902 Sophia Antipolis Cedex, France. e-mail: \\ jean-baptiste.pometesophia.inria.fr
}

Keywords: River modelling, Time delay systems, Nonlinear models, Internal Model Control

\begin{abstract}
A simple nonlinear model for flow transfer in a river stretch is obtained through several steps: first the diffusive wave model, a quasilinear partial derivative equation, is linearized around equilibrium points, corresponding to the hydraulic equilibrium regimes. Secondly, the moment matching method is used to approximate the linear PDE by a linear ODE with a time delay indexed by the value of the reference discharge. A single nonlinear model is then derived analytically from this family of linear models. The proposed model can reproduce the nonlinear behavior of the time-delay with discharge variations. This simple nonlinear model is inserted into a Internal Model Controller framework in order to obtain a nonlinear controller to control river flow. Simulations demonstrates the performance of the nonlinear controller compared to a linear one.
\end{abstract}

\section{Introduction}

Automatic control is considered as an possible effective means to improve water management of dam-river systems (where a dam is used to control the discharge at some point in the river downstream). In this perspective, it is important to have accurate models of flow transfer. This introduction presents the possible interests of the paper first for the presented application (water management) then in a more general view for control theory.

Modelling of the flow routing in a river stretch has been the subject of numerous publications since the 1950's. Weinman and Laurenson [12] presented a comprehensive review of approximate flow routing methods and show that all available methods are derived from either kinematic wave or diffusion analogy models.

Qualitatively, one of the important (nonlinear) feature of the behavior of a river stretch is the presence of a time-delay (induced by the propagation phenomenon) that depends on the discharge $^{1}$ with important variations, especially for low discharges.

Different linear models have been developed for simulation

\footnotetext{
${ }^{1}$ The discharge is the quantity of water through a vertical section per unit of time.
}

purposes $[10,4]$. Their main limitation is their narrow domain of validity: they are only valid if the discharge remains close to the point of linearization. Other nonlinear models were also developed to replicate the nonlinear feature of flow transfer in a river [2]; however, they incorporate a constant time-delay, which cannot match correctly the full PDE model.

Some nonlinear models have been recently developed, which represent correctly the time-delay variation with the discharge, but these models were designed for simulation purposes, and are not described in a manner that would allow controller design. For example, Camacho and Lees [3] used a close approach to the one presented here, but the model obtained is of fractional order, and the order changes as the discharge varies. Such a model cannot be used to design a controller with classical nonlinear control methods.

In the present article, we obtain a "simple" nonlinear model for the discharge transfer in a river from a knowledge-based model (diffusive wave model). It has fixed order, but contains a variable time-delay. This model should be adapted to the case of rivers with low flows, where the delay varies a lot with the discharge. An appealing feature of a model is the fact that its parameters are directly related to channel characteristics and hydraulic conditions. Simplicity also enables calculation in limited time, which gives perspectives in terms of utilization of the model in real-time, for example for forecasting.

The final objective of this work is the design of strategies to control the flow along the river via a dam located upstream. This problem has mainly been considered in the linear context [11]. A first attempt to use the obtained nonlinear model is presented at the end of the paper, with its inclusion in an Internal Model Controller framework, to deal with the variable time-delay. The proposed nonlinear controller leads to a better performance than a similar linear one.

The nonlinear model is obtained through several steps. Section 2 briefly presents the control problem. Then, the diffusive wave model, a quasilinear partial derivative equation, is linearized around equilibrium points, corresponding to the hydraulic permanent regimes. This linear PDE is then approximated by a linear ODE with delay using the moment matching method, which leads to a family of linear models indexed by the value of the constant discharge around which they are valid. These two steps are carried out in section 3 .

A single nonlinear model is then derived analytically from 
these linear models; around each linearization point, it coincides with the corresponding linear model. This can be seen as the main result of the paper, and is presented in section 4 .

Section 5 is devoted to validate the obtained nonlinear model, by comparing it, through simulations, to the initial diffusive wave model; it turns out to be able to adequately reproduce the nonlinear behavior of the time-delay with discharge variations.

We end, in section 6, with a first control application of the proposed model: we design a nonlinear IMC controller using this nonlinear model to control the flow in a river. This controller is stable for a large range of discharges and leads to a better performance than a linear one.

Notations: The acronyms ODE and PDE stand respectively for Ordinary Differential Equation and Partial Differential Equation and IMC stands for Internal Model Control.

\section{Control problem}

The system considered in this paper is a controlled river where the action variable is the upstream discharge and the measured variable is the downstream discharge (see figure 1). The river is used to deliver water from the upstream dam to various consumers pumping water along the river (farmers irrigating their fields, industries, etc.). The objective of the controller is to keep the measured downstream discharge close to a target despite unmeasured users' withdrawals.

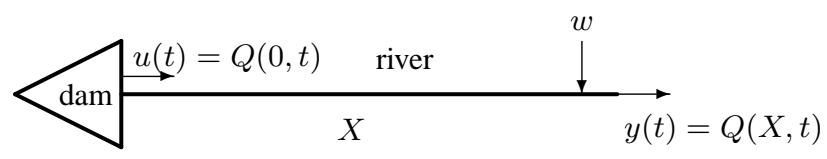

Figure 1: Dam-river system considered

In other terms, the control objective is to use the upstream discharge $u$ in order to keep the downstream discharge $y$ as constant as possible, which means that the control should attenuate the unmeasured perturbations $w$. This is a problem of regulation or desensitivity.

We now focus on the modelling part.

\section{Obtaining a family of linear ODEs from a quasilinear PDE}

\subsection{The quasilinear PDE: diffusive wave model}

The diffusive wave equation is a quasilinear partial differential equation obtained by simplification of Saint-Venant model [7]:

$$
\frac{\partial Q}{\partial t}+\Theta(Q) \frac{\partial Q}{\partial x}-E(Q) \frac{\partial^{2} Q}{\partial x^{2}}=0
$$

with $Q(x, t)$ the discharge $\left(\mathrm{m}^{3} / \mathrm{s}\right), \Theta(Q)$ the celerity $(\mathrm{m} / \mathrm{s})$ and $E(Q, x)$ the diffusion $\left(\mathrm{m}^{2} / \mathrm{s}\right)$.

The boundary conditions are given by $Q(0, t)=u(t)$, $\lim _{x \rightarrow \infty} \frac{\partial Q(x, t)}{\partial x}=0$. The controlled output is $y(t)=Q(X, t)$, where $X$ is the length of the river stretch.

$\Theta(Q, x)$ and $E(Q, x)$ have relatively complex expressions in the general case, but simpler expressions are available for specific river geometry. Assuming uniform geometry, and if we neglect the effect of backwater curves, $\Theta$ and $E$ can be expressed as functions of the discharge $Q$. In particular for a rectangular large uniform geometry (slope $I$, width $L$, Manning friction coefficient $n), \Theta$ and $E$ are then given by:

$$
\begin{aligned}
\Theta(Q) & =\frac{5 I^{0.3} Q^{0.4}}{3 L^{0.4} n^{0.6}} \\
E(Q) & =\frac{Q}{2 L I}
\end{aligned}
$$

The above expressions can be replaced by more complicated functions of $Q$ (for example functions obtained by identification in case of a more realistic geometry [5]), without changing the method exposed below.

\subsection{The linear PDE: Hayami model}

The linearization of equation (1) around a constant reference discharge $Q_{e} \neq 0\left(Q=Q_{e}+\delta Q\right)$ gives the Hayami equation:

$$
\frac{\partial \delta Q}{\partial t}+\Theta_{e} \frac{\partial \delta Q}{\partial x}-E_{e} \frac{\partial^{2} \delta Q}{\partial x^{2}}=0
$$

with $\Theta_{e}=\Theta\left(Q_{e}\right)$ and $E_{e}=E\left(Q_{e}\right)$.

Considering the boundary conditions $u(t)=\delta Q(0, t)$ and $\lim _{x \rightarrow \infty} \frac{\partial \delta Q}{\partial x}=0$, and the measured output $y(t)=\delta Q(X, t)$, the linear PDE (4) with can be represented by an irrational transfer function in the Laplace domain. It is the Hayami transfer function, relating upstream and downstream discharge variations $y(s)=F_{\text {Hayami }}(s) u(s)$, where:

$$
F_{\text {Hayami }}(s)=e^{\frac{\Theta_{e}-\sqrt{\Theta_{e}^{2}+4 E_{e} s}}{2 E_{e}}} X
$$

with $s$ the Laplace variable $\left[\mathrm{s}^{-1}\right]$.

\subsection{Approximation of the linear PDE by a linear ODE}

Inverse Laplace transform tables give the impulse response of Hayami transfer function (5):

$$
\mathcal{L}^{-1}\left[F_{\text {Hayami }}(s)\right]=\frac{X}{2 \sqrt{\pi E_{e} t^{3}}} e^{-\frac{\left(X-\Theta_{e} t\right)^{2}}{4 E_{e} t}}
$$

It is very close to the one of a second order with delay (see figure 2):

$$
F_{2 r}(s)=\frac{G e^{-s \tau}}{1+S s+P s^{2}}
$$




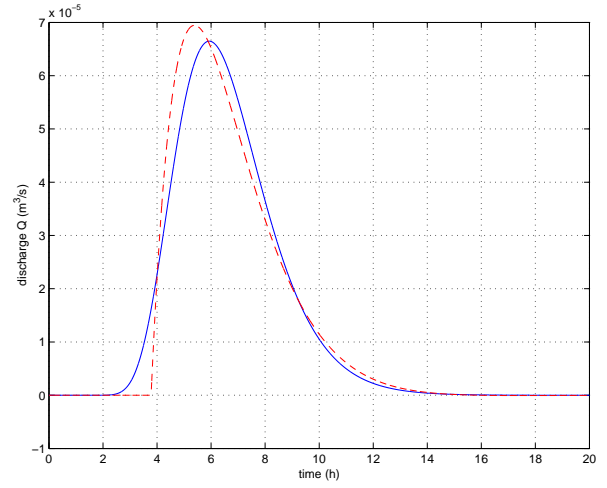

Figure 2: Impulse responses of Hayami transfer function (5) $(-)$ and of the second order with delay (7) $(--)$

We use the moment matching method to fit the frequency response of the transfer functions (5) and (7).

The purpose of the moment matching method is to equate the low order moments of two different models. It is a way to reproduce the low frequency behavior of a given transfer function, which is the main frequency range encountered in natural systems. This method is used to identify the four moments of the transfer function $F_{2 r}(s)$ to those of the Hayami transfer function.

Equating the first four impulse moments of both transfer functions and solving for the unknowns $S, P$ and $\tau$ leads to an polynomial equation of degree 3 , that can be solved analytically [8]. Following a hydraulic classification of rivers [1], the non-dimensional coefficient $\chi=\frac{3 X \Theta_{e}}{10 E_{e}}$ characterizes the behavior of the river for flow transfer. It is assumed in the following that $\chi>1.35$, which leads to identifying a stable second order transfer function with delay (see [8]). In this case, the parameters of the second order transfer function with delay are given by:

$$
\begin{aligned}
G & =1 \\
\tau & =\frac{X}{\Theta_{e}}-S \\
S & =2 \sqrt{\frac{2 X E_{e}}{\Theta_{e}^{3}}} \cos (\phi / 3) \\
P & =\frac{2 X E_{e}}{\Theta_{e}^{3}}\left(1-\frac{3 E_{e}}{S \Theta_{e}^{2}}\right) \\
\phi & =\frac{\pi}{2}+\arctan \sqrt{\frac{9 E_{e}}{2 X \Theta_{e}-9 E_{e}}}
\end{aligned}
$$

It should be noted that in the majority of cases, rivers are in the range $\chi>1.35$, and are therefore well represented by a second order with delay model.

\section{Obtaining a nonlinear ODE from a family of linear ODEs}

We are now in the situation where we have a family of linear models, indexed by the value $Q_{e}$ of the (constant) discharge around which they are valid. A natural question to ask is whether it is possible to find a single nonlinear system that would have these linear systems as linear approximation at a suitable family of operating points.

The problem can be posed slightly more generally as follows : given a family of delay differential systems $\left(\Sigma \mathcal{L}_{\lambda}\right)_{\lambda \in \mathbb{R}}$ indexed by a scalar parameter $\lambda$ :

$$
\Sigma \mathcal{L}_{\lambda}\left\{\begin{array}{l}
\dot{X}(t)=A(\lambda) X(t)+B(\lambda) U(t) \\
Y(t)=C(\lambda) X(t-\tau(\lambda)),
\end{array}\right.
$$

does there exist a nonlinear system

$$
\begin{aligned}
& \dot{\xi}(t)=f(\xi(t), u(t)) \\
& \eta(t)=h(\xi(t-\sigma(\xi)))
\end{aligned}
$$

and a curve $\lambda \mapsto \xi_{e}^{\lambda}$ of equilibria, i.e.

$$
f\left(\xi_{e}^{\lambda}, u_{e}^{\lambda}\right)=0
$$

such that each system $\Sigma \mathcal{L}_{\lambda}$ be the linearized system of (14) around $\left(\xi_{e}^{\lambda}, u_{e}^{\lambda}\right)$ ?

This question is difficult in the general case. It is addressed here in the case of the linear systems obtained in section 3, after considering the problem of linearization of a system with variable delay.

\subsection{Linearization of a system with variable delay}

We restrict the analysis to the case of an equilibrium point, i.e. $\bar{\xi}$ and $\bar{u}$ are constant and $f(\bar{\xi}, \bar{u})=0$.

Lemma 1 Consider an equilibrium point $f(\bar{\xi}, \bar{u})=0$ and the stable time invariant linear system with delay

$$
\begin{aligned}
\delta \dot{\xi} & =A \delta \xi+B \delta u \\
\delta \eta(t) & =C \delta \xi(t-\sigma(\bar{\xi}))
\end{aligned}
$$

with

$$
A=\frac{\partial f}{\partial x}(\bar{\xi}, \bar{u}), \quad B=\frac{\partial f}{\partial u}(\bar{\xi}, \bar{u}), \quad C=\frac{\partial h}{\partial x}(\bar{\xi})
$$

Then the output $\eta(t)$ of the control system (14) starting at $\bar{\xi}$ with control $t \mapsto \bar{u}+\delta u(t)$ is given by

$$
\eta(t)=h(\bar{\zeta}(t-\sigma(\bar{\zeta}(t))))+\delta \eta(t)
$$

where $\delta \eta(t)$ is the output of (16) starting at 0 with control $\delta u(t)$. 


\subsection{Application to the family of linear models obtained in section 3.3}

The model obtained by the moment matching method from the model of Hayami has parameters that depend explicitly on the reference discharge $Q_{e}$, since $\Theta_{e}$ and $E_{e}$ depend on $Q_{e}$. We can therefore apply the results of section 4.

The flow transfer in the river is now represented by a second order with delay transfer function $F_{2 r}(s)=\frac{e^{-\tau s}}{1+S s+P s^{2}}$ with $\tau, S$ and $P$ given by the equations (9-12). Choosing $\delta x=$ $[\dot{\delta y}, \delta y]^{T}$ as state variables for the system without delay, this linear system can be represented in state space by:

$$
\left\{\begin{array}{l}
\dot{\delta x}(t)=A(\lambda) \delta x(t)+B(\lambda) \delta u(t) \\
\delta y(t)=C(\lambda) \delta x(t-\tau(\lambda))
\end{array}\right.
$$

with $A(\lambda)=\left(\begin{array}{cc}-\frac{S(\lambda)}{P(\lambda)} & -\frac{1}{P(\lambda)} \\ 1 & 0\end{array}\right), B(\lambda)=\left(\begin{array}{c}\frac{1}{P(\lambda)} \\ 0\end{array}\right)$, $C(\lambda)=\left(\begin{array}{cc}0 & 1\end{array}\right)$ and $\lambda=Q_{e}$.

Lemma 2 Consider the nonlinear system described by

$$
\left\{\begin{array}{l}
\dot{x}(t)=A\left(x_{2}(t)\right) x(t)+B\left(x_{2}(t)\right) u(t) \\
y(t)=x_{2}\left(t-\tau\left(x_{2}(t)\right)\right)
\end{array}\right.
$$

with $x=\left(x_{1} x_{2}\right)^{T}, A\left(x_{2}\right)$ and $B\left(x_{2}\right)$ given by equations above, $\tau\left(x_{2}\right)=\frac{X}{\Theta\left(x_{2}\right)}-S\left(x_{2}\right)$, $S\left(x_{2}\right)=2 \sqrt{\frac{2 X E\left(x_{2}\right)}{\Theta\left(x_{2}\right)^{3}}} \cos \left(\phi\left(x_{2}\right) / 3\right), \quad P\left(x_{2}\right)=$ $\frac{2 X E\left(x_{2}\right)}{\Theta\left(x_{2}\right)^{3}}\left(1-\frac{3 E\left(x_{2}\right)}{\Theta\left(x_{2}\right)^{2} S\left(x_{2}\right)}\right), \quad \phi\left(x_{2}\right) \quad=\quad \frac{\pi}{2} \quad+$ $\arctan \sqrt{\frac{9 E\left(x_{2}\right)}{2 X \Theta\left(x_{2}\right)-9 E\left(x_{2}\right)}}, \Theta(x)$ and $E(x)$ given by equations (2) and (3), the initial state given by $x_{0}(t)=\left[\begin{array}{ll}0 & Q_{0}(t)\end{array}\right]^{T}$, for $t \in\left[-\tau_{\max }, 0\right]$, where $\tau_{\max }$ is the maximum value of the delay, obtained in our case for the minimum value of the input discharge.

Then each linearization of (21) around an equilibrium trajectory $u_{e}=x_{2 e}=Q_{e}$ coincides with the linear system (20) where $\lambda=Q_{e}$.

Proof: The linearization of (21) around $\left(x_{e}, u_{e}\right)$ leads to :

$$
\frac{\partial f}{\partial x}\left(x_{e}, u_{e}\right)=\left(\begin{array}{cc}
a_{11} & a_{12} \\
1 & 0
\end{array}\right)
$$

with $a_{11}=-\frac{S\left(x_{2 e}\right)}{P\left(x_{2 e}\right)}$ and $a_{12}=-\frac{1}{P\left(x_{2 e}\right)}+\left(u_{e}-x_{2 e}\right) \frac{P^{\prime}\left(x_{2 e}\right)}{P^{2}\left(x_{2 e}\right)}-$ $x_{1 e} \frac{S^{\prime}\left(x_{2 e}\right) P\left(x_{2 e}\right)-S\left(x_{2 e}\right) P^{\prime}\left(x_{2 e}\right)}{P^{2}\left(x_{2 e}\right)}$. Since $u_{2}=x_{2 e}$ and $x_{1 e}=0$, one recovers the expression (20) for $x_{2 e}=\lambda$. We also have:

$$
\frac{\partial f}{\partial u}\left(x_{e}, u_{e}\right)=\left(\begin{array}{c}
\frac{1}{P\left(x_{2 e}\right)} \\
0
\end{array}\right)
$$

The other terms are obtained in the same way.

Remark: This model is only valid for slow variations of the state $x$. It is also an underlying hypothesis for the diffusive wave model. If the discharge varies very quickly, the celerity and the diffusion depending on the discharge would certainly give results different from physical observations. Indeed, the diffusive wave equation is obtained by neglecting inertia terms in the dynamic equation of Saint-Venant's model [9]. These inertia terms contain $\frac{\partial Q}{\partial t}$, which is not negligible if the discharge varies quickly.

\section{Nonlinear model validation}

The nonlinear model with delay obtained is rather particular, as the output delay depends on the state of the non delayed system. We will first give the conditions on $\tau$ such that the system is well-posed, then we will compare it in simulation with the original PDE nonlinear model.

\subsection{Well-posedness}

The observation equation of the nonlinear system with delay (21) is well-posed if and only if

$$
\tau\left(x_{2}(t)\right)<t
$$

which implies

$$
\dot{\tau}<1
$$

This can be written

$$
x_{1}(t) \tau^{\prime}\left(x_{2}(t)\right)<1
$$

where $\tau^{\prime}=\frac{d \tau(x)}{d x}$.

The condition (22) can be checked at each time $t$.

\subsection{Numerical implementation}

The system being composed of a nonlinear system with a delay in output, the nonlinear system without delay is first solved with a Runge-Kutta method of order 4, and the delayed output is computed in a second step.

\subsection{Nonlinear model simulations}

Validation is done by comparing the output of the model proposed with the one of the diffusive wave model for the same input data. The diffusive wave equation is solved using a semiimplicit finite difference scheme (Crank-Nicholson scheme). We also compare the output of the proposed model to the one given by the linear Hayami model obtained by linearization around the initial discharge $u(0)$. The computations are done for a river of length $X=10 \mathrm{~km}$, width $L=8 \mathrm{~m}$, Manning $n=0.05$, slope $I=0.0004$.

The nonlinear model with delay reproduces well the behavior of the river, and is very close to the diffusive wave model (see figure 3). One notices that the main characteristic of these rivers for low flows, i.e. the variation of the delay with the discharge, is taken well into account by the nonlinear model with delay, whereas this is not the case for the linear model. 

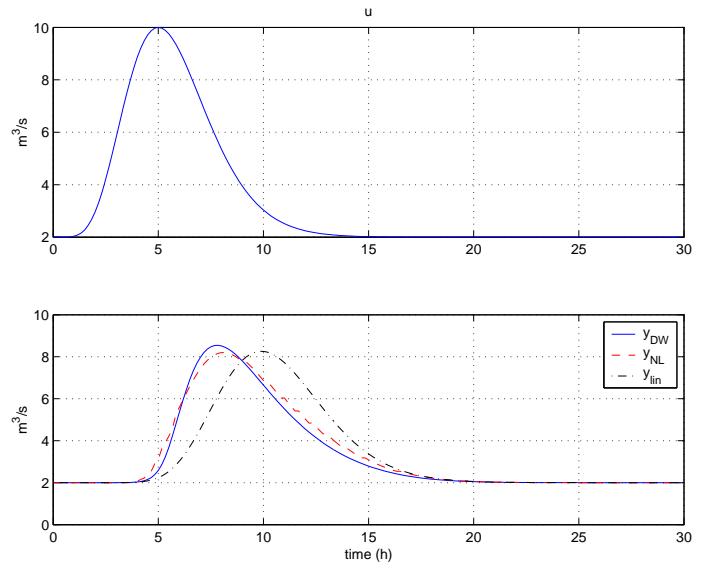

Figure 3: Comparison between the diffusive wave model $\left(y_{D W}\right.$ $-)$, the nonlinear model of second order with delay $\left(y_{N L}--\right)$ and the linear PDE model obtained for $Q_{e}=u(0)\left(y_{l i n}-.-\right)$

\section{Nonlinear IMC controller design}

The proposed model is used to design a nonlinear IMC controller for the dam-river system. Adding an additive output perturbation $w$ to the model (representing water withdrawals), leads to:

$$
\left\{\begin{array}{l}
\dot{x}(t)=A\left(x_{2}(t)\right) x(t)+B\left(x_{2}(t)\right) u(t) \\
y(t)=x_{2}\left(t-\tau\left(x_{2}(t)\right)\right)+w(t)
\end{array}\right.
$$

The control objectives as stated in section 2 are then twofold:

- reject unknown output perturbations $w$

- track a reference signal $y_{c}$

This should be valid on a large discharge range, since the released discharge changes a lot during the irrigation season.

\subsection{Nonlinear IMC controller design}

The design is inspired from the Internal Model Controller already developed in a linear framework [6].

The IMC based architecture enables an independent estimation of withdrawal $w$. The closed-loop command is obtained by comparing the measured output $y$ to the predicted output of the internal model $\hat{y}$. The difference represents the internal model estimation of unpredicted withdrawal $\hat{w}$. This estimation is filtered by a linear filter $f(s)$. The filter is designed to compensate for the discrepancies between the predicted value of perturbations $\hat{w}$ and the actual one $w$, due to model uncertainties. If the nominal model was a perfect description of the system, the effect of the withdrawal would be exactly accounted for by the control scheme and the predicted value would be perfectly accurate.

Once a model is chosen, the choice of controller parameter is then reduced to the choice of the robustness filter. For simplic-

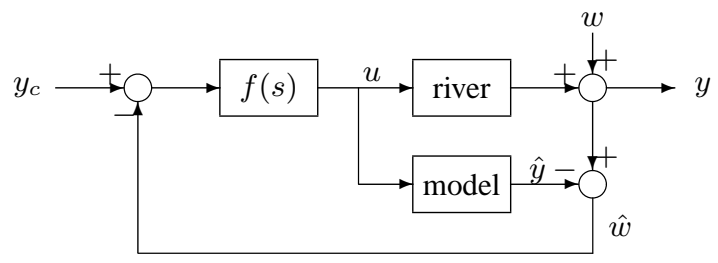

Figure 4: IMC controller architecture

ity, we chose a first order filter $f(s)$, so there is only one parameter to tune, the filter time constant. This constant is evaluated with the linear robust design procedure described in [6]. This leads to a rather smooth linear controller, which is robust stable for large variations of the reference discharge. The use of a nonlinear model instead of a linear one is expected to increase the performance of the controlled system, since model errors should be reduced. This procedure can be extended to the case of intermediate discharge measurements [6] (in this case, the system becomes SIMO).

It should be noted that the time-delay of the system varies from $43 \mathrm{~min}$ for $Q_{e}=10 \mathrm{~m}^{3} / \mathrm{s}$ to $227 \mathrm{~min}$ for $Q_{e}=1 \mathrm{~m}^{3} / \mathrm{s}$. The filter obtained for the linear controller is a first order filter $f(s)=\frac{1}{1+T s}$ with time constant $T=195 \mathrm{~min}$. For the nonlinear controller, the filter is chosen equal to 1 (no filtering).

\subsection{Simulations}

The controlled system is simulated with the original quasilinear PDE over a large range of discharges, with unknown perturbations and reference changes at various operating points. The figure 5 shows the ability of the nonlinear IMC controller to reject unknown perturbations and track reference, compared to a linear IMC controller. The use of a nonlinear model enables to greatly improve the real-time performance: the nonlinear controller reacts from 4 to 13 hours quicker than the linear one. This can have important implications in terms of water management. This increase in real-time performance is mainly due to a better estimation of perturbations. As a matter of fact, the error in estimated perturbations $\hat{w}$ is much lower for the nonlinear controller (see figure 6). The important error in the linear case necessitates a strong filtering in order to guarantee stability for a wide range of time-delays. This leads to a degradation of the real-time performance, which can be significantly enhanced with a nonlinear model.

\section{Conclusion}

We described in this paper a way to obtain a simple ODE nonlinear model from a quasilinear PDE. After linearization around a reference regime, the moment matching method is applied to the linear PDE in order to get an approximation of its irrational transfer function. Then a single nonlinear model 

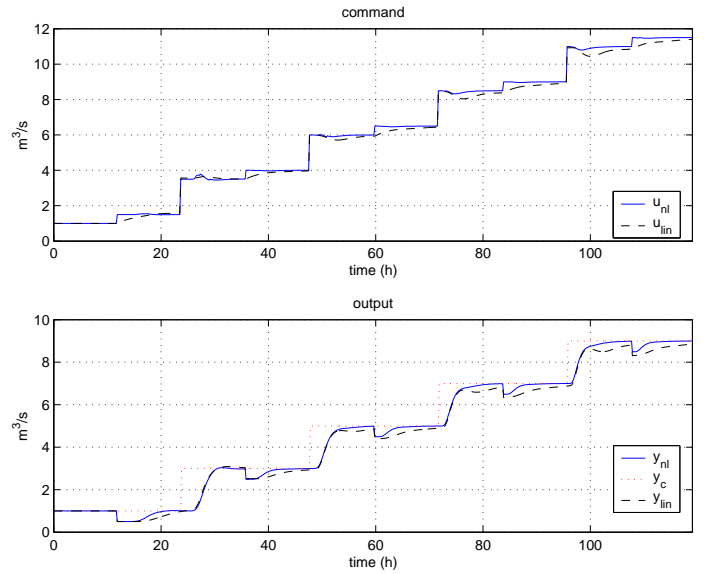

Figure 5: Simulation of the closed-loop system: comparison between the nonlinear IMC controller (-) and the linear IMC controller $(--)$

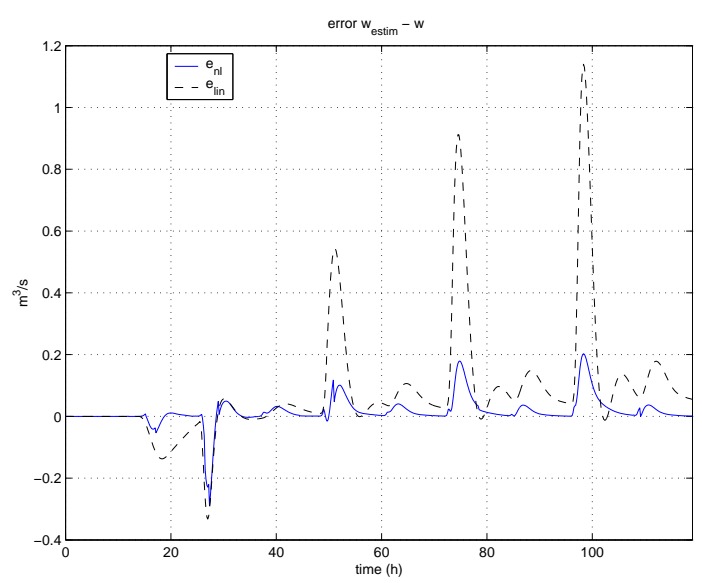

Figure 6: Error in estimated perturbations $\hat{w}$ : comparison between the nonlinear IMC controller (-) and the linear IMC controller $(--)$

is obtained from the family of linear ones indexed by the value of the discharge around which they were linearized.

The obtained nonlinear model has the advantage to be directly related to physical parameters, as the length of the river stretch $X$, the slope $I$, Manning coefficient $n$ and the width $L$. This model can be used for many different purposes:

- For quick simulation purposes, as it is easier to implement than a complete numerical resolution of the initial partial differential equation,

- For identification purposes: a model-based identification usually necessitates numerous simulations of the model, which is time-consuming. A simpler model can be simulated more quickly,

- For controller design, as some dams located upstream can be used to control the discharge downstream of the river.
We used the proposed model to design a nonlinear IMC controller. It has been tested in simulation with the quasilinear PDE, and appeared to be able to function on a wide range of discharges. The use of a nonlinear model enables to significantly increase the performance of a linear IMC controller.

\section{Acknowledgements}

This work was initiated when the first author was occupying a post-doctorate position at INRIA Sophia Antipolis with MIAOU Project, under grant provided by INRIA Scientific Direction.

The first author wishes to thank Vincent Fromion for useful comments on the paper.

\section{References}

[1] J.P. Baume, J. Sau, and P.-O. Malaterre. Modeling of irrigation channel dynamics for controller design. In Conf. on Systems, Man and Cybernetics, SMC'98, pages 3856-3861, San Diego, 1998.

[2] P. L. F. Bentura and C. Michel. Flood routing in a wide channel with a quadratic lag-and-route method. Hydrological Sciences, 42(2):169-189, 1997.

[3] L.A. Camacho and M.J. Lees. Multilinear discrete lag-cascade model for channel routing. J. of Hydrology, 226:30-47, 1999.

[4] J.A. Cunge. On the subject of a flood propagation computation method (Muskingum method). J. of Hydraulic Research, 7(2):205-230, 1969.

[5] X. Litrico. Nonlinear diffusive wave modeling and identification for open-channels. J. of Hydraulic Engineering, 127(4):313$320,2001$.

[6] X. Litrico. Robust IMC flow control of SIMO dam-river openchannel systems. IEEE Trans. on Control Systems Technology, 10(3):432-437, 2002.

[7] X. Litrico and D. Georges. Robust continuous-time and discretetime flow control of a dam-river system (I): Modelling. Applied Mathematical Modelling, 23(11):809-827, 1999.

[8] P.-O. Malaterre. Modélisation, analyse et commande optimale LQR d'un canal d'irrigation. Ph.D. thesis, ISBN 2-85362-3688, Etude EEE ${ }^{\circ}$. 14, LAAS - CNRS - ENGREF - Cemagref, 1994. (in French).

[9] W.A. Miller and J.A. Cunge. Simplified equations of unsteady flow, pages 183-257. Water Resources Publications, Fort Collins, Colorado, 1975.

[10] R. Moussa. Analytical Hayami solution for the diffusive wave flood routing problem with lateral inflow. Hydrological Processes, 10:1209-1227, 1996.

[11] M. Papageorgiou and A. Messmer. Flow control of a long river stretch. Automatica, 25(2):177-183, 1989.

[12] P.E. Weinmann and E.M. Laurenson. Approximate flood routing methods: A review. J. of the Hydraulic Division, December 1979. 